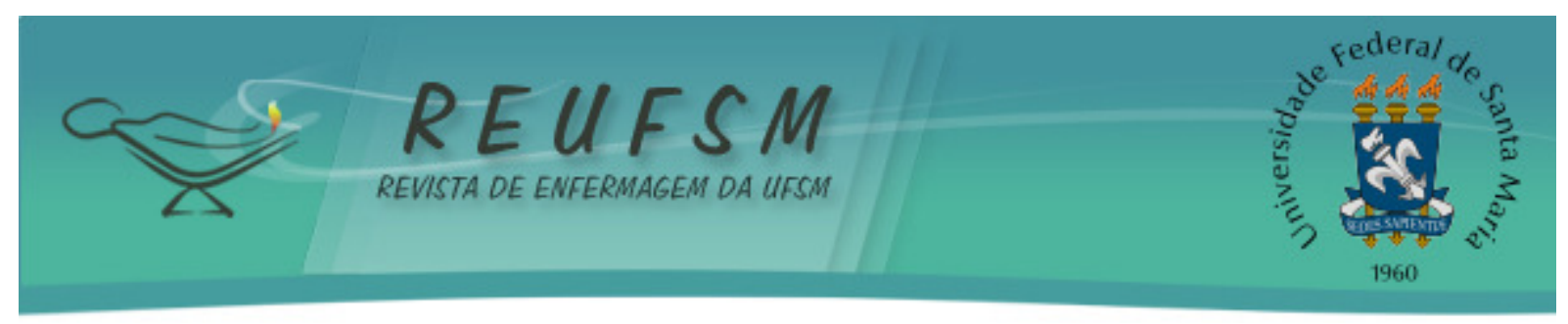

\title{
DIAGNÓSTICOS DE ENFERMAGEM NO PÓS-OPERATÓRIO IMEDIATO DE CIRURGIA DE TROCA DE VÁLVULA
}

\section{NURSING DIAGNOSIS IN THE IMMEDIATE POSTOPERATIVE OF VALVE REPLACEMENT SURGERY \\ DIAGNÓSTICO DE ENFERMERÍA EN EL POSTQUIRÚRGICO INMEDIATO DE LA CIRUGÍA DE SUSTITUCIÓN DE VÁLVULA}

\author{
Bruna Fontes de Lara ${ }^{1}$ \\ Paula Cristina Nogueira ${ }^{2}$ \\ Vanessa de Brito Poveda ${ }^{3}$
}

Doi: $10.5902 / 2179769225716$

RESUMO: Objetivo: identificar os diagnósticos de enfermagem no pós-operatório imediato de cirurgia de troca de válvula. Método: estudo documental, de corte transversal, realizado por meio dos prontuários de pacientes submetidos à cirurgias valvares entre janeiro e dezembro de 2014, em um hospital de grande porte, especializado em cardiologia, do Estado de São Paulo. Resultados: entre os 50 prontuários analisados, $58 \%$ pacientes eram do sexo feminino. Foram identificados 11 diagnósticos de enfermagem, sendo $55 \%$ classificados com foco no problema e $45 \%$ de risco. Os diagnósticos de enfermagem mais prevalentes foram Risco de Infecção (100\%), Mobilidade Física Prejudicada (94\%) e Risco de Queda (94\%). Conclusão: o diagnóstico mais prevalente no pós-operatório imediato de cirurgia foi risco de infecção. Os diagnósticos identificados possibilitaram a compreensão mais adequada da experiência vivenciada pelo paciente e a percepção dos enfermeiros em relação ao processo saúde doença do mesmo, contribuindo para a otimização de recursos humanos e materiais.

Descritores: Cirurgia torácica; Anuloplastia da valva cardíaca; Diagnóstico de enfermagem.

ABSTRACT: Aim: To identify the nursing diagnoses in the immediate postoperative period of valve replacement surgery. Method: Documental and cross-sectional study carried out by means of the medical records of patients submitted to valve surgeries between January and December 2014, in a large hospital specialized in cardiology in the state of São Paulo. Results: among the 50 analyzed charts, $58 \%$ belonged to female patients. Eleven nursing diagnoses were identified, 55\% are classified by the health problem and $45 \%$ are risk. The most prevalent nursing diagnoses were Infection Risk (100\%), Impaired Physical Mobility (94\%) and Risk of Fall (94\%). Conclusion: the highest occurrence diagnosis identified in the immediate postoperative period of surgery was the risk of infection. The identified diagnoses allowed a better understanding of the experience lived by the patient and the nurses' perception of it, contributing to the optimization of human and material resources.

Descriptors: Thoracic surgery; Cardiac valve annuloplasty; Nursing diagnosis.

\footnotetext{
${ }^{1}$ Enfermeira. Especialista em Enfermagem Cardiovascular pela Escola de Enfermagem da Universidade de São Paulo e Instituto Dante Pazzanese de Cardiologia. São Paulo, SP, Brasil. E-mail: brunafonteslara@gmail.com

${ }^{2}$ Enfermeira. Pós-doutora em Ciências. Professor Doutor do Departamento de Enfermagem Médico-Cirúrgica da Escola de Enfermagem da Universidade de São Paulo. São Paulo, SP, Brasil. E-mail: pcnogueira@usp.br

3 Enfermeira. Pós-doutora em Enfermagem. Professor Doutor do Departamento de Enfermagem MédicoCirúrgica da Escola de Enfermagem da Universidade de São Paulo. São Paulo, SP, Brasil. E-mail: vbpoveda@usp.br
} 


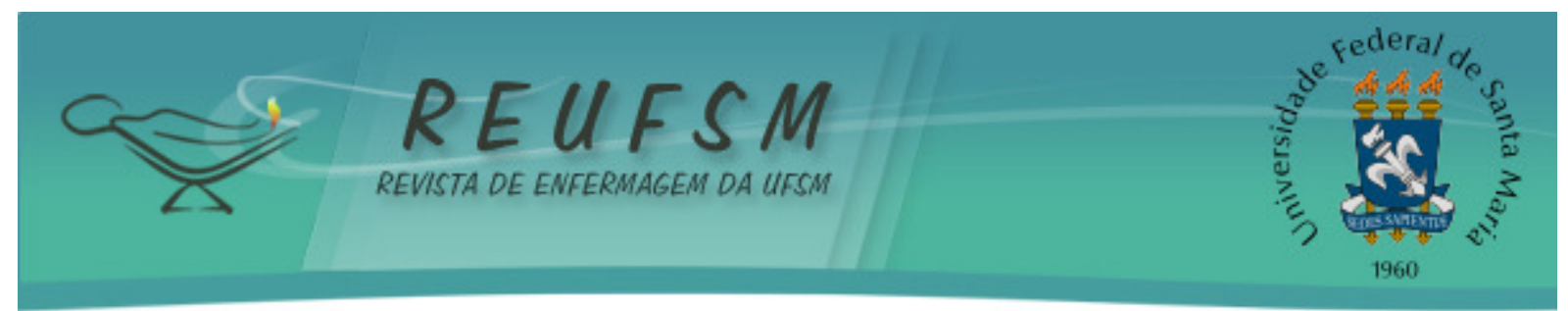

RESUMEN: Objetivo: identificar los diagnósticos de enfermería en el postquirúrgico inmediato de la cirugía de sustitución de la válvula. Método: estudio documental, de recorte transversal, realizado a partir de las historias clínicas de los pacientes sometidos a cirugías valvulares entre enero y diciembre de 2014, en un hospital grande, especializado en cardiología, de la provincia de São Paulo. Resultados: entre las 50 historias analizadas $58 \%$ eran mujeres. Fueron identificados 11 diagnósticos de enfermería, de esos 55\% están clasificados por el problema y $45 \%$ por el riesgo. Los diagnósticos de enfermería más prevalentes fueron Riesgo de Infección (100\%), Dificultad de Movilidad Física (94\%) y Riesgo de Caídas (94\%). Conclusión: el diagnóstico del postoperatorio inmediato de mayor ocurrencia fue el riesgo de infección. Los diagnósticos identificados posibilitaron la comprensión más adecuada de la experiencia vivida por el paciente y la percepción de los enfermeros en relación al proceso salud enfermedad de ese sujeto, contribuyendo para la optimización de los recursos humanos y materiales.

Descriptores: Cirugía torácica; Anuloplastia de la válvula cardíaca; Diagnóstico de enfermería.

\section{INTRODUÇÃO}

Mundialmente, as doenças cardiovasculares são consideradas a principal causa de morte e queda da qualidade de vida relacionada à incapacidade. Embora tenha ocorrido um declínio em suas taxas de mortalidade nas últimas décadas, continua presente o impacto das doenças cardiovasculares no sistema de saúde brasileiro sendo que, em 2011, as doenças do aparelho circulatório foram responsáveis por 335.213 mortes. ${ }^{1}$

Portanto, as doenças cardiovasculares impactam o Sistema Único de Saúde (SUS), gerando a necessidade de novas propostas relacionadas a intervenções estratégicas efetivas em saúde para minimizar a expressão das doenças cardiovasculares no Brasil.

Entre as doenças cardiovasculares destacam-se as doenças isquêmicas do coração, as cerebrovasculares, a insuficiência cardíaca e valvulopatias que, apesar de constituírem as principais causas de morbidade e mortalidade no Brasil e no mundo, podem ser tratadas clinica, hemodinâmica e cirurgicamente. ${ }^{2}$

As doenças valvares são subdivididas em insuficiência ou estenose e promovem alterações nos ventrículos, acarretando disfunções diastólicas ou sistólicas, que progridem para a insuficiência cardíaca. Especialmente, as insuficiências podem ocasionar disfunção ventricular importante, provocando uma indicação cirúrgica precoce, ou seja, antes mesmo do aparecimento dos sintomas. Já as lesões estenóticas podem aguardar até o aparecimento dos sintomas para uma indicação cirúrgica, no entanto, existe controvérsia 


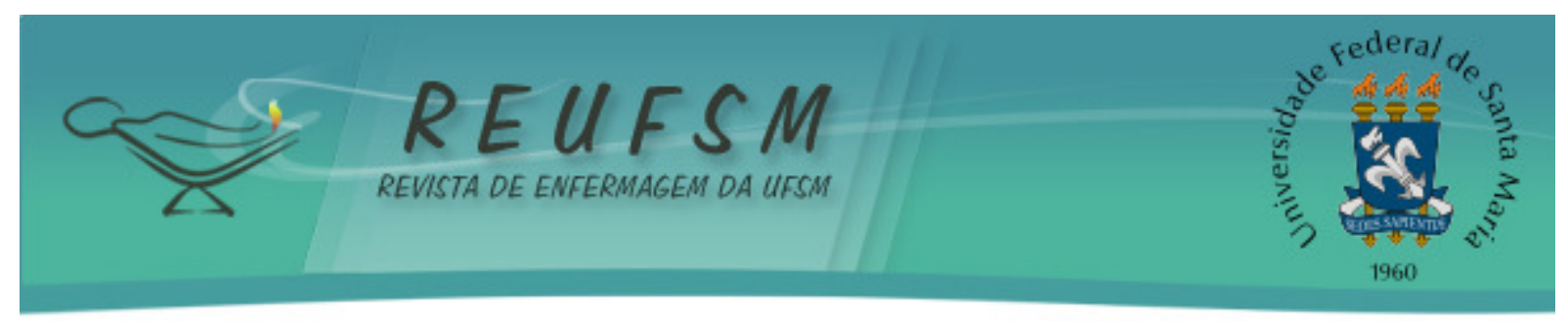

sobre o momento correto da indicação do procedimento cirúrgico em indivíduos assintomáticos. $^{3}$

As cirurgias valvares consistem na troca da valva inativa por prótese mecânica ou biológica. Nos Estados Unidos da América (EUA) a cirurgia de troca de valva aórtica, corresponde a $13 \%$ de todas as cirurgias cardíacas em adultos, sendo que no Reino Unido é a segunda cirurgia cardíaca mais comum. ${ }^{4}$ No Brasil, a cirurgia valvar envolvendo a troca da válvula cardíaca por uma prótese mecânica ou biológica, constitui a segunda mais frequente e representa 17,4\% das cirurgias cardiovasculares, realizadas entre os anos de 2008 a 2010, ${ }^{4}$ destacando-se que o tratamento das valvulopatias também pode ser realizado clinicamente, por meio de medicamentos.

A prótese biológica, proveniente de tecidos porcinos ou bovinos remontados em anéis é a mais utilizada, por dispensar o uso contínuo de anticoagulantes e indicada, principalmente, em pacientes idosos. Sua limitação está relacionada à durabilidade, a necessidade de reoperações e a calcificação, o que acarreta aumento do risco cirúrgico em longo prazo. ${ }^{3}$ Já as próteses mecânicas são produzidas através de um processo industrial do carvão pirolítico, não apresentando desgaste ao longo do tempo e, por este motivo, indicadas para adultos jovens e crianças. Faz-se necessário controle rigoroso de coagulação, devido a necessidade permanente de anticoagulação. ${ }^{3}$

Neste sentido, os cuidados de enfermagem são imprescindíveis para a recuperação do paciente submetido a esta categoria de procedimentos cirúrgicos. Diante desse cenário, por meio da Sistematização da Assistência de Enfermagem (SAE), a enfermagem tem se aperfeiçoado e implementado novas estratégias de assistência, individualizadas e que atendam o cliente, sua família e comunidade. ${ }^{5}$

O enfermeiro realiza ações de cuidado nas diferentes fases que compõem o período perioperatório, em especial no pós-operatório imediato, que engloba as primeiras 24 horas a partir da conclusão do procedimento anestésico-cirúrgico. Inclui, portanto, o tempo de sala de recuperação pós-anestésica, no qual a equipe de enfermagem atua na avaliação constante de consciência e restabelecimento da normalidade de sinais vitais. ${ }^{7}$

Diante da complexidade de cuidados requeridos por pacientes com afecções valvares que precisam de intervenções de enfermagem imediatas, os diagnósticos de enfermagem possibilitam o estabelecimento de um cuidado individualizado, com foco nas respostas dos 


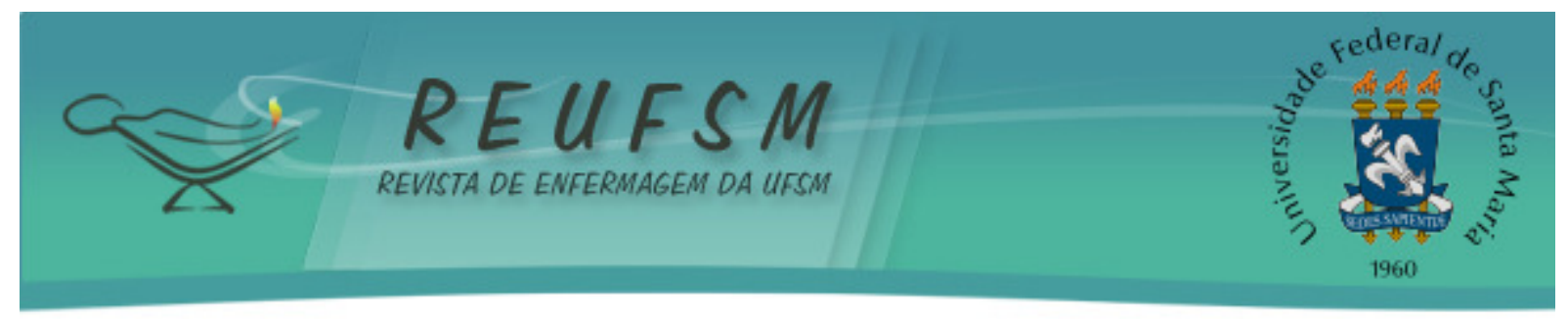

pacientes aos problemas de saúde vivenciados. Assim, orientam o estabelecimento de ações de enfermagem mais eficientes na resolução das necessidades identificadas.

Desse modo, estudos que possibilitam compreender a experiência vivenciada pelo paciente e a percepção dos enfermeiros em relação ao processo saúde doença vivenciado pelo paciente, propiciam o direcionamento de recursos humanos e materiais, contribuindo para a qualidade da assistência prestada e a construção do conhecimento em enfermagem, em qualquer nível de assistência á saúde.

Portanto, este estudo teve como finalidade identificar os diagnósticos de enfermagem, mais frequentes nos pós-operatório imediato de cirurgia de troca de válvula, colaborando para a estruturação de um plano de cuidado, individualizado e consonante com a clínica de cada paciente, garantindo a prevenção de complicações e proporcionando o retorno às atividades cotidianas. Assim, este estudo objetivou identificar os diagnósticos de enfermagem no pósoperatório imediato de troca de válvula.

\section{MÉTODO}

Trata-se de um estudo documental, de corte transversal, realizado por meio de prontuários de pacientes submetidos à cirurgia de troca de válvula, entre janeiro e dezembro de 2014, em um hospital de grande porte do Sudeste de São Paulo.

Foram incluídos pacientes de ambos os sexos, com idade $\geq 18$ anos, submetidos à cirurgia de troca de válvula, entre janeiro e dezembro de 2014. Foram excluídos pacientes que foram a óbito durante a internação ou submetidos a múltiplos procedimentos no período da pesquisa.

Os dados foram coletados por meio dos registros realizados no pós-operatório imediato pela equipe de enfermagem, contidos nos prontuários dos pacientes. Para os pacientes que tiveram mais de um atendimento no período, foi considerado o último registro de enfermagem, o qual foi armazenado no serviço de arquivo médico (SAME).

A coleta se deu no período de outubro de 2015 à janeiro de 2016, utilizando-se um instrumento de coleta que continha informações a respeito das variáveis sociodemográficas e clínicas, além dos diagnósticos de enfermagem realizados no pós-operatório imediato pelos enfermeiros assistenciais e as intercorrências apresentadas no pós-operatório. 


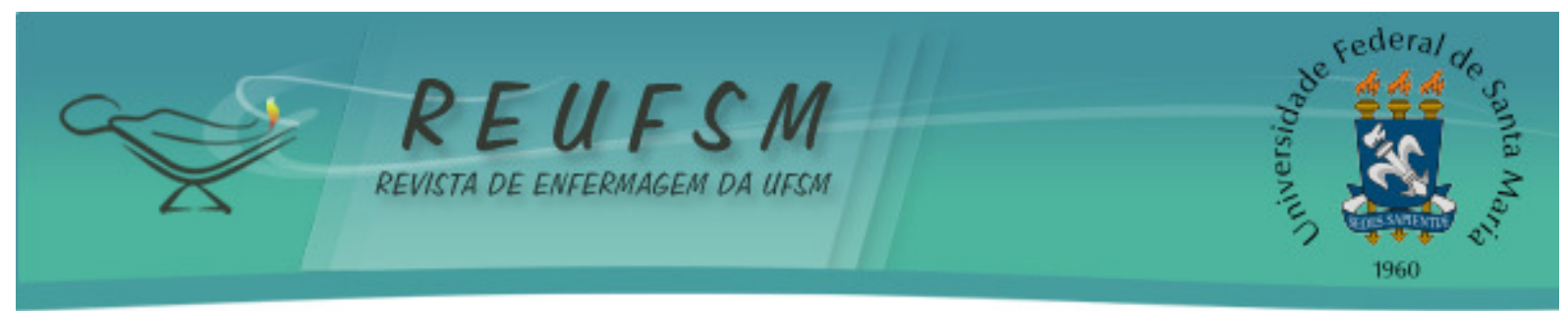

Os dados foram digitados em uma planilha do Excel e analisados pelo programa software SPSS. As variáveis categóricas foram analisadas utilizando o teste Qui-Quadrado. O nível de significância delimitado foi de $\alpha<0,05$.

Os princípios éticos foram respeitados em relação ao acesso e análise dos dados. Este estudo foi analisado e aprovado pelo Comitê de Ética e Pesquisa com Seres Humanos, sob CAAE: 49638115400005462 e parecer $n^{\circ} 4608$.

\section{RESULTADOS}

Foram analisados 50 prontuários. A maioria dos pacientes eram do sexo feminino (58\%), com idade média de 55,5 anos, dos quais $60 \%$ viviam com seus respectivos companheiros. As demais características clínicas são apresentadas na tabela 1 .

Tabela 1 - Perfil sociodemográfico e clínico dos pacientes submetidos a cirurgias valvares. São Paulo/SP, 2016

\begin{tabular}{|c|c|c|}
\hline Variavéis & $\mathbf{n}$ & $\%$ \\
\hline \multicolumn{3}{|l|}{ Sexo } \\
\hline Masculino & 21 & 42 \\
\hline Feminino & 29 & 58 \\
\hline \multicolumn{3}{|l|}{ Estado Civil } \\
\hline Casado & 32 & 64 \\
\hline Solteiro & 9 & 18 \\
\hline Viúvo & 8 & 16 \\
\hline Divorciado & 1 & 2 \\
\hline \multicolumn{3}{|l|}{ Idade } \\
\hline $20-29$ & 1 & 2 \\
\hline $30-39$ & 4 & 8 \\
\hline $40-49$ & 10 & 20 \\
\hline $50-59$ & 14 & 28 \\
\hline$\geq 60$ & 21 & 42 \\
\hline \multicolumn{3}{|l|}{ Comorbidades } \\
\hline Hipertensão Arterial & 31 & 62 \\
\hline Dislipidemia & 13 & 26 \\
\hline Diabetes Melittus & 11 & 22 \\
\hline Tabagismo & 4 & 8 \\
\hline Sedentarismo & 2 & 4 \\
\hline Etilista & 1 & 2 \\
\hline
\end{tabular}

A insuficiência cardíaca foi o diagnóstico médico de maior prevalência acometendo 29 dos pacientes $(58 \%)$; na sequência, a disfunção de prótese $(10,20 \%)$; dupla lesão $(9 ; 18 \%)$; estenose (4;8\%); e outros eventos $(4 ; 8 \%)$. 


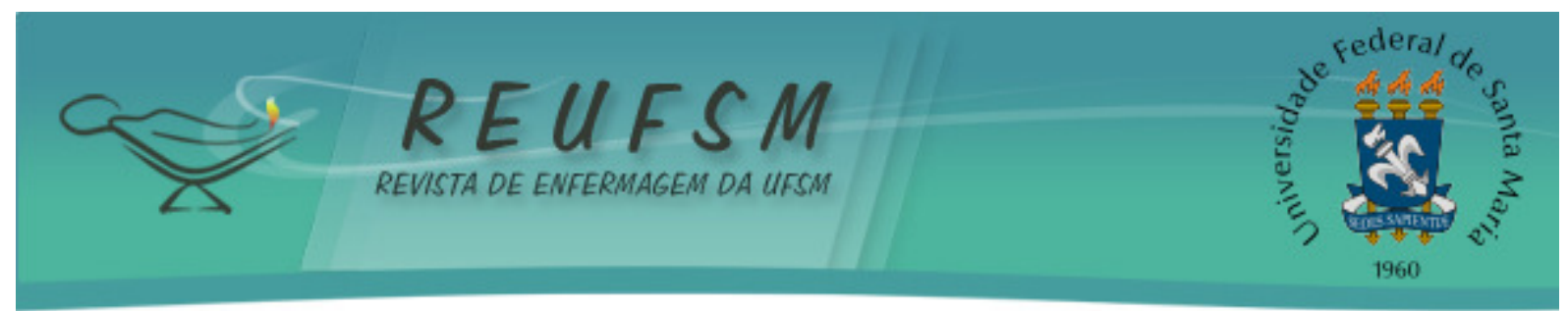

A cirurgia mais frequente foi troca de válvula em 47 dos casos (94\%), seguida por plastia ou retroca $(2 ; 4 \%)$. O tipo de válvula mais utilizada foi a prótese biológica $(31 ; 62 \%)$.

Considerando os casos estudados, foram identificados 11 diagnósticos de enfermagem (DE) diferentes, sendo que o Risco de Infecção foi observado em 100\% da amostra estudada, seguido por mobilidade física prejudicada (94\%) e risco de queda (94\%) (Tabela 2).

Tabela 2 - Diagnósticos de enfermagem identificados no pós-operatório imediato de troca de válvula. São Paulo/SP, 2016.

\begin{tabular}{lcc}
\hline Diagnósticos & n & \% \\
\hline Risco de Volume de Líquido Deficiente & 41 & 82 \\
Mobilidade Física Prejudicada & 47 & 94 \\
Débito Cardíaco Diminuído & 32 & 64 \\
Comunicação Verbal Prejudicada & 28 & 56 \\
Processos Familiares Interrompidos & 28 & 56 \\
Risco de Infecção & 50 & 100 \\
Risco de Queda & 47 & 94 \\
Risco de Integridade da Pele Prejudicada & 46 & 92 \\
Integridade Tissular Prejudicada & 44 & 88 \\
Risco de Aspiração & 36 & 72 \\
Hipotermia & 10 & 20 \\
\hline
\end{tabular}

Observou-se que dos 11 diagnósticos de enfermagem, seis (55\%) eram classificados como DE com foco no problema, entre eles, mobilidade física prejudicada; integridade tissular prejudicada; débito cardíaco diminuído; processos familiares interrompidos; comunicação verbal prejudicada e hipotermia. Cinco (45\%) eram diagnósticos de risco, entre eles, risco de infecção; risco de queda; risco para integridade da pele prejudicada; risco de volume de líquidos deficientes; e risco de aspiração (Quadro 1). Quanto aos domínios predominantes, destacaram-se os relacionados à segurança e proteção $(6 ; 54,5 \%)$, seguido pelo domínio atividade e repouso $(2 ; 18,1 \%)$. Os domínios nutrição, percepção e cognição e papéis e relacionamentos apresentaram apenas um diagnóstico (9\%) cada (Tabela 2).

Os resultados das associações entre diagnósticos de enfermagem e as variáveis sociodemográficas e clínico-cirúrgicas estão descritas no Quadro 1. 


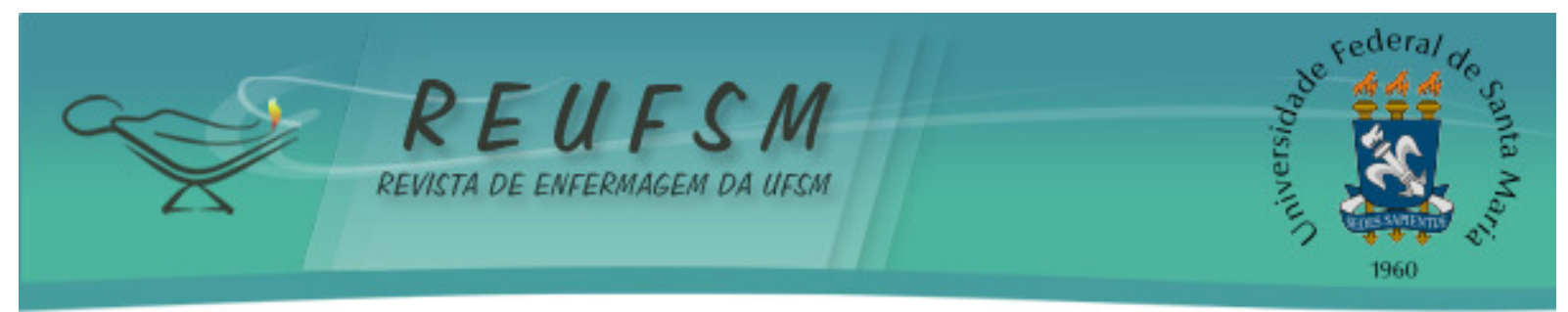

Quadro 1 - Associações estatisticamente significantes entre diagnósticos de enfermagem e variáveis sociodemográficas e clínico-cirúrgicas. São Paulo/SP, 2016.

\begin{tabular}{|c|c|c|c|c|}
\hline Diagnósticos de Enfermagem & Variáveis & $\mathbf{n}$ & $\%$ & $\mathbf{p}^{*}$ \\
\hline Risco de Aspiração & Sexo Masculino & 21 & 42 & 0,013 \\
\hline \multirow[t]{3}{*}{ Comunicação Verbal Prejudicada } & Estenose Mitral & 3 & 6 & 0,046 \\
\hline & Sangramento & 5 & 10 & 0,046 \\
\hline & Hipertensão Arterial Sistêmica & 31 & 62 & 0,033 \\
\hline \multirow[t]{4}{*}{ Processos familiares interrompidos } & Cirurgia Cardíaca Prévia & 1 & 2 & 0,007 \\
\hline & Estenose Mitral & 3 & 6 & 0,046 \\
\hline & Sangramento & 5 & 10 & 0,046 \\
\hline & Hipertensão Arterial Sistêmica & 31 & 62 & 0,033 \\
\hline \multirow{2}{*}{ Hipotermia } & Fibrilação Ventricular & 1 & 2 & 0,046 \\
\hline & Hipotermia & 10 & 20 & 0,046 \\
\hline Risco de Queda & Insuficiência Renal Aguda & 2 & 4 & 0,008 \\
\hline Mobilidade física prejudicada & Trombose Aguda de Prótese & 2 & 4 & 0,008 \\
\hline \multirow[t]{3}{*}{ Integridade tissular prejudicada } & Edema Agudo de Pulmão & 1 & 2 & 0,007 \\
\hline & Tabagista & 4 & 8 & 0,028 \\
\hline & Hipertensão Arterial Sistêmica & 31 & 62 & 0,007 \\
\hline
\end{tabular}

* Valores estatisticamente significantes pelo Teste Qui - Quadrado

\section{DISCUSSÃO}

Observou-se, nesta investigação, a preferência do serviço quanto ao uso de próteses do tipo biológica, o que pode estar associado à idade dos indivíduos, uma vez que o estudo mostrou um predomínio de indivíduos entre 60 a 69 anos. A idade superior a 65 anos é um dos critérios para indicação deste material, uma vez que a prótese metálica deve ser utilizada em pacientes com expectativa de longa sobrevida devido a sua maior durabilidade. ${ }^{8}$

Embora, mesmo após 30 anos da introdução de próteses modernas, a escolha entre próteses biológicas ou mecânicas mantem-se controversa, pela carência de estudos científicos de qualidade metodológica e/ou com grande número de sujeitos que determinem a melhor forma de tratamento destas afecções. Por esse motivo, em geral, os médicos realizam recomendações embasadas na experiência clínica e no bom senso. ${ }^{8}$

Tem sido observado na literatura científica, o predomínio de mulheres idosas com comorbidades variadas, submetidas a cirurgias cardíacas, em especial troca de válvula. ${ }^{9-11} \mathrm{O}$ conhecimento das variavéis sociodemográficas, clínicas e cirúrgicas dos indivíduos submetidos à cirurgia cardíaca são essenciais para o planejamento de qualidade da assistência de enfermagem. ${ }^{5,11-13}$ Tendo em vista que diversos destes aspectos podem contribuir para aumentar o tempo de permanência em Unidade de Terapia Intensiva (UTI) e tempo de ventilação mecânica. $^{11,14-15}$ 


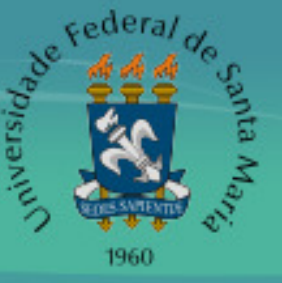

Estudo recente demonstrou que o diabetes mellitus (DM) é um preditor para tempo prolongado de UTI, ou seja, pacientes diabéticos apresentam maior chance de permanecerem internados nestas unidades, por um maior período de tempo, em relação aos pacientes não diabéticos. ${ }^{14}$

Além disso, diversos aspectos como DM, HAS, tabagismo, dislipidemia e idade avançada, estão associados ao aumento da necessidade do uso de ventilação mecânica ou ventilação mecânica prolongada $(\mathrm{VM}){ }^{15}$

Assim, pacientes tabagistas que não suspenderam o cigarro até quatro semanas antes do procedimento cirúrgico, podem apresentar maior permanência na UTI, devido à dificuldade existente na adequada oxigenação, exigindo maior tempo em ventilação mecânica. $^{11}$ Da mesma forma, pacientes dislipidêmicos e com idade avançada podem apresentar tempo de ventilação mecânica $\leq 48$ horas. ${ }^{1,16}$

Deste modo, é possível observar que, hábitos de vida, comorbidades préexistentes e a idade dos pacientes podem interferir no prognóstico dos sujeitos submetidos à cirurgia de troca de válvula. Assim, os diagnósticos de enfermagem apresentados no pósoperatório imediato são marcados, principalmente, por diagnósticos com foco no problema e dos domínios segurança e proteção e atividade e repouso.

Destaca-se também, a associação entre os DE e as variáveis clínico-cirúrgicas e complicações pós-operatórias. Os resultados mostram associação entre o DE integridade tissular e a ocorrência de edema agudo de pulmão. A literatura científica recente demonstrou que o edema agudo de pulmão é um preditor para VM prolongada, e portanto, resulta em tempo prolongado de restrição no leito. Além disso, este DE também está associado ao tabagismo, que como citado requer VM prolongada, com maior restrição ao leito e aumento do tempo de internação hospitalar. Da mesma forma, a cirurgia cardíaca prévia é um fator independente para VM prolongada, o que resulta em maior tempo de permanência do paciente no leito. ${ }^{9-10,17}$

Assim, estudos anteriores realizados com pacientes no pós-operatório de cirurgia cardíaca elencaram diagnósticos iguais aos do presente estudo, entre eles: mobilidade física prejudicada, débito cardíaco diminuído, hipotermia, comunicação verbal prejudicada, risco de integridade da pele prejudicada, risco de queda, risco de aspiração, risco de infecção e integridade tissular prejudicada., ${ }^{5,18}$ 


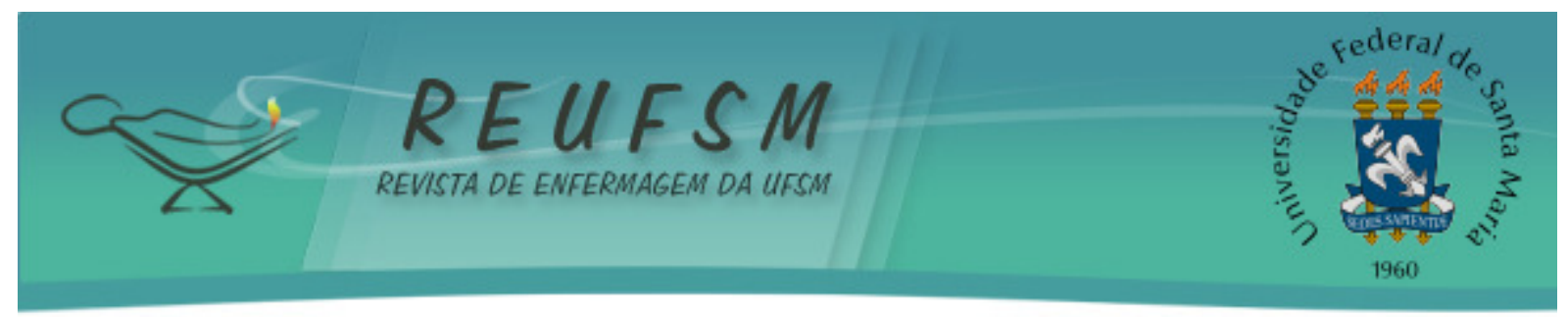

O diagnóstico Risco de Infecção tem alta prevalência em pacientes submetidos à cirurgia cardíaca, possivelmente, relacionado à necessidade de procedimentos invasivos, como por exemplo: cateterismo vesical, acesso venoso central, drenos de tórax e mediastino, acesso arterial para pressão arterial média, além da destruição dos tecidos e das defesas primárias. ${ }^{5}$

Da mesma forma, o diagnóstico Mobilidade Física Prejudicada ocorre com frequência em virtude da restrição no leito, decorrente do procedimento cirúrgico e da utilização de drenos, que restringem a movimentação do corpo, além da sensação de dor durante o movimento. ${ }^{5,18}$

Interessante destacar a associação entre o diagnóstico de enfermagem (DE) risco de aspiração e o sexo masculino, que também foi observado em investigação prévia sobre os preditores de ventilação mecânica prolongada em pacientes submetidos à cirurgia de válvula.

O diagnóstico médico Estenose Mitral é caracterizado pelo espessamento e imobilidade dos folhetos valvares, ocasionando resistência ao fluxo sanguíneo através da válvula. Tal patologia quando não tratada de maneira adequada, pode acarretar diversas complicações como dispnéia, edema pulmonar, embolia arterial sistêmica, contribuindo para o DE comunicação verbal prejudicada associado ao desconforto respiratório, e, sobretudo, mantendo o paciente na unidade de terapia intensiva UTI, justificando o DE processos familiares interrompidos. ${ }^{19}$

O DE comunicação verbal prejudicada e processos familiares interrompidos estiveram associados à hipertensão arterial. Estudos mostram que pacientes com história de HAS no pré-operatório permanecem intubados por mais tempo, o que dificulta e/ou impossibilita a comunicação do individuo, aumenta o tempo de permanência na UTI, resulta no afastamento do paciente e sua família e também contribui para o DE integridade tissular prejudicada, uma vez que este paciente fica mais tempo restrito ao leito. ${ }^{11,15-16}$

Importante destacar a associação entre os DE comunicação verbal prejudicada, processos familiares interrompidos, débito cardíaco diminuído e a intercorrência sangramento. O sangramento é considerado a segunda intercorrência mais frequente, acometendo em torno de $10 \%$ dos casos. Interessante frisar que o sangramento altera a funcionalidade do aparelho circulatório, especialmente a pré e pós-carga, contribuindo para diminuição do débito cardíaco, aumentando o tempo de permanência na UTI. ${ }^{12,20}$

O DE hipotermia esteve associada à ocorrência de hipotermia em recuperação pósanestésica. Estudos apontam que essa condição acomete de 60\% a $90 \%$ dos pacientes submetidos à anestesia e está relacionado aos efeitos das drogas anestésicas sobre a 


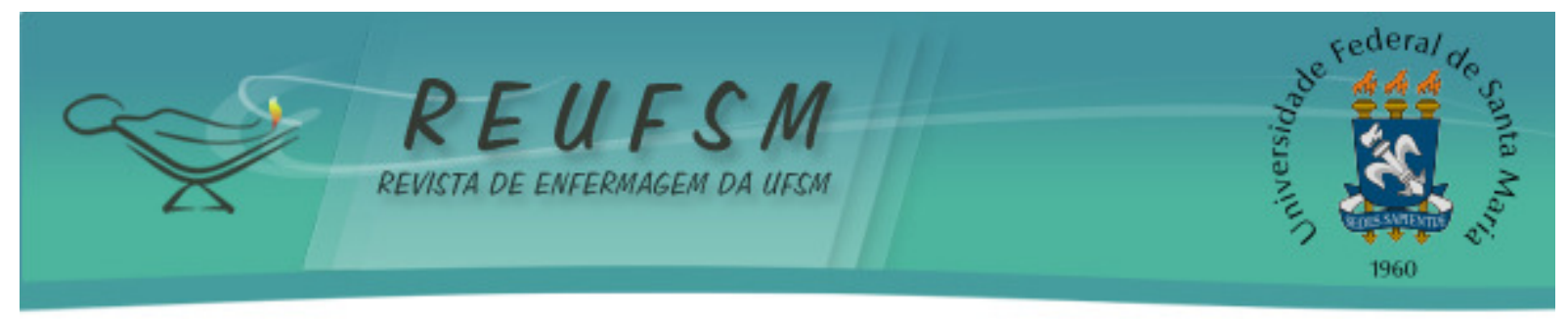

termorregulação e redução do metabolismo, além de outros aspectos como a exposição do paciente a temperatura fria na sala cirúrgica e o uso de circulação extracorpórea. ${ }^{20}$

A insuficiência renal aguda (IRA) pode ocorrer como uma intercorrência no pósoperatório. Estudos recentes mostram que idade avançada é um fator de risco para o desenvolvimento de IRA. ${ }^{21-22}$ Além disso, idosos apresentam maior incidência de quedas, devido à perda da massa muscular, ligada há uma serie de disfunções e doenças sistêmicas comuns no indivíduo idoso. ${ }^{21-22}$

O DE mobilidade física prejudicada foi associado à intercorrência trombose aguda de prótese. Pacientes que apresentam trombose aguda de prótese necessitam de restrição ao leito, devido ao risco de deslocamento do trombo e possível embolia pulmonar. ${ }^{23}$

Assim, os resultados do presente estudo possibilitam compreender como o procedimento cirúrgico e todas as repercussões advindas dele refletem em aspectos clínicos e de vida pessoal e como estas situações afetam o cuidado de enfermagem. Além disso, a identificação precoce dos acontecimentos favorece a prevenção de complicações. Todos estes aspectos associados contribuem para qualidade da assistência prestada e a construção do conhecimento em enfermagem na área de especialidade.

\section{CONCLUSÃO}

Este estudo permitiu identificar os diagnósticos de enfermagem no pós-operatório imediato de cirurgia de troca de válvula. Observou-se o predomínio de mulheres, idosas, com insuficiência cardíaca, submetidas à cirurgia de troca de válvula por prótese biológica e que apresentavam comorbidades como hipertensão arterial sistêmica, diabetes mellitus e dislipidemia.

Em relação aos diagnósticos de enfermagem identificados no pós-operatório imediato de cirurgia de válvula pertencem aos domínios segurança e proteção e atividade e repouso. $\mathrm{O}$ diagnóstico de maior prevalência na população estudada foi risco de infecção.

Nota-se que, apesar dos diagnósticos de enfermagem no pós-operatório imediato de cirurgia de troca de válvula estarem bem descritos e/ou elencados, publicações de enfermeiros que estabeleçam associações entre seus achados à clínica dos pacientes ainda são escassas.

Os resultados do presente estudo podem colaborar com os enfermeiros na determinação dos eventos mais frequentes entre pacientes submetidos a cirurgias valvulares, permitindo um planejamento adequado de recursos humanos e materiais. 


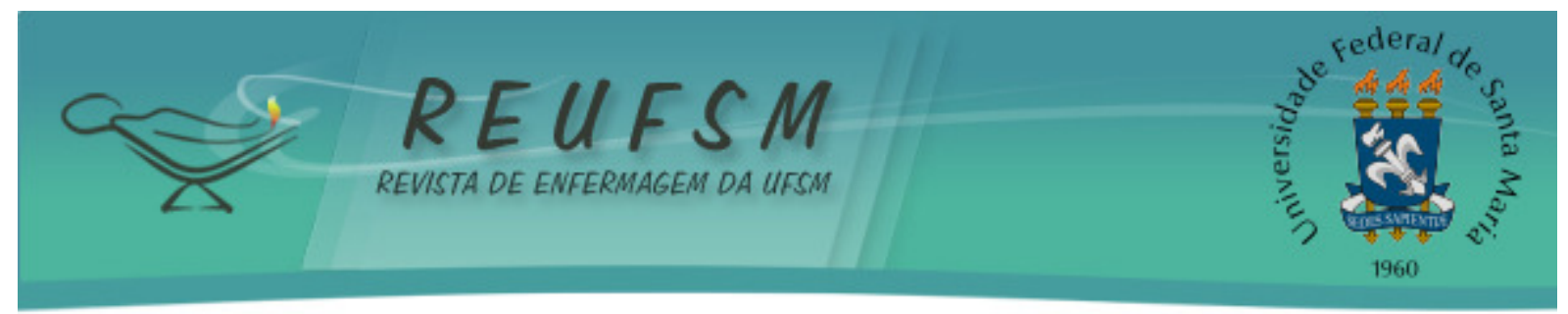

\section{REFERÊNCIAS}

1. Datasus. Indicadores de mortalidade [Internet]. 2012 [acesso 12 abr 2017]. Disponível em: http://tabnet.datasus.gov.br/cgi/deftohtm.exe?idb2012/c08.def.

2. Assis CC, Lopes JL, Martins LAN, Barros ALBL. Acolhimento e sintomas de ansiedade em pacientes no pré - operatório de cirurgia cardíaca. Rev Bras Enferm. 2014;67(3):401-7.

3. Caputo PMS. Avaliação neurológica e da função cognitiva em pacientes com alto risco de embolização submetidos á cirurgia de troca valvar aórtica por estenose calcificada: papel do filtro intra - aórtica [dissertação]. Porto Alegre: Universidade Federal do Rio Grande do Sul, Faculdade de Medicina de Porto Alegre; 2013. 59 p.

4. Ribeiro FFF, Arruda GDAS, Ptak DM, Menezes FR, Torrico MAO, Ramos AIO. Seguimento pós - operatório em longo prazo de pacientes submetidos á cirurgia valvar. Rev Bras Cardiol. 2013;26(2):112-9.

5. Ribeiro CP, Silveira CO, Benetti ERR, Gomes JS, Stumm EMF. Diagnósticos de enfermagem em pacientes no pós - operatório de cirurgia cardíaca. Rev Rene. 2015;16(2):159-67.

6. Moreira RAN, Caetano JA, Barros LM, Galvão MTG. Diagnóstico de enfermagem, fatores relacionados e de risco no pós - operatório de cirurgia bariátrica. Rev Esc Enferm USP. 2013;47(1):168-75.

7. Bertoncello KCG, Sávio B, Ferreira JM. Diagnósticos e propostas de intervenções de enfermagem aos pacientes em pós-operatório imediato de cirurgia eletiva. Cogitare Enferm. 2014;19(3):582-9.

8. Beccaria LM, Cesarino CB, Werneck AL, Correio NCG, Correio SS, Correio MNM. Complicações pós - operatórias em pacientes submetidos á cirurgia cardíaca em hospital de ensino. Arq Ciênc Saúde. 2015;3(22):37-41.

9. Shirzad M, Karimi A, Ahmadi SH, Marzban M, Tazik M, Aramin H. Predictors and early outcome of prolonged mechanical ventilation in contemporary heart valve surgery. Monaldi Arch Chest Dis. 2010;74(1):22-7.

10. Araujo NR, Araújo RA, Oliveira RC, Bezerra SMMS. Complicações pós-operatórias em pacientes submetidos à cirurgia de revascularização miocárdica. Rev Enferm UFPE. 2013;7(5):1301-10.

11. Laizo A, Delgado EF, Rocha GM. Complicações que aumentam o tempo de permanência na unidade de terapia intensiva na cirurgia cardíaca. Rev Bras Cir Cardiovasc. 2010;25(2):166-77.

12. Silveira CR, Santos MBKS, Moraes MAP, Souza EN. Desfechos clínicos de pacientes submetidos á cirurgia cardíaca em um hospital do nordeste do Rio Grande do Sul. Rev Enferm UFSM. 2016;6(1):102-11.

13. Bastos AS, Beccaria LM, Barbosa TP, Werneck AL, Silva EV. Complications in patients after percutaneous aortic valve replacement. Acta Paul Enferm. 2016;29(3):267-73.

14. Oliveira EK, Turquetto ALR, Tauil PL, Junqueira Junior LF, Porto GG. Risk factors for prolonged hospital stay after isolated coronary artery bypass grafting. Rev Bras Cir Cardiovasc. 2013;28(3):353-63. 


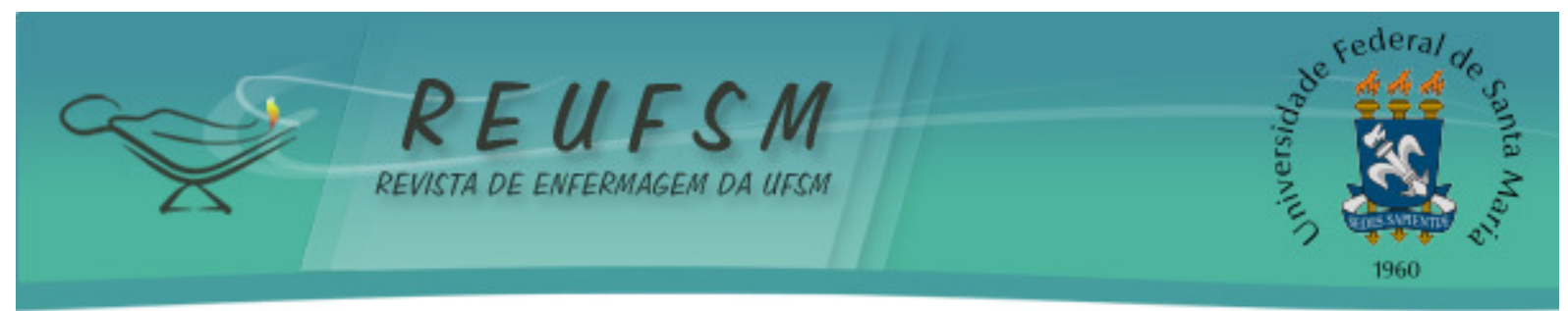

15. Siddiqui MMA, Paras I, Jalal A. Risk factors of prolonged mechanical ventilation following open heart surgery: what has changed over the last decade? Cardiovas Diagn Ther. 2012;2(3):192-9.

16. Abdulrdha MF, Tawfiq NB. Impact of preoperative risk factors upon duration of intubation for cardiosurgery patients in Ibn-al-bettarcenter. Kufa Journal For Nursing Sciences. 2015;5(2):1-9.

17. Piotto RF, Ferreira FB, Colósimo FC, Silveira GS, Sousa AG, Braile DM. Independent predictors of prolonged mechanical ventilation after coronary artery bypass surgery. Rev Bras Cir Cardiovasc. 2012;27(4):520-8.

18. Nakasato GR, Lopes CT, Barros ALBL. Diagnóstico de enfermagem no perioperatório de cirurgia cardíaca. Rev Min Enferm. 2015;19(4):980-6.

19. Almeida MR. Apresentação clínica da estenose mitral em pacientes com cardiopatia reumática no hospital universitário professor Edgard Santos, Salvador, Bahia [monografia]. Salvador: Faculdade de Medicina da Bahia, da Universidade Federal da Bahia; 2016.

20. Branco CSP, Pereira HO. Cuidados de enfermagem ao paciente em pós - operatório imediato de cirurgia de revascularização do miocárdio. Enferm Rev. 2016;19(1):72-84.

21. Magro MC, Zerbato B, Gonçalves DM. Rifle e Akin: Impacto na predição da injúria renal aguda em pós-operatório de cirurgia cardíaca. Rev Cubana Enferm. 2015;31(3).

22. Kalsing A, Oliveira GG, Silva IS, Neris JCD, Knorst R. Análise de fatores de risco de queda em idosos internados em um hospital terciário no sul do Brasil. Rev Bras Ciênc Envelhecimento Humano (RBCEH). 2016;13(1):48-60.

23. Campos NLKL. Comparison of the occurrence of thromboembolic and bleeding complications in patients with mechanical heart valve prosthesis with one and two leaflets in the mitral position. Rev Bras Cir Cardiovasc. 2014;29(1):59-61.

Data de submissão: 26/01/2017

Data de aceite: 09/11/2017

Autor correspondente: Bruna Fontes de Lara

Endereço: Rua Rio das Antas, 145, Jardim Santo Antônio

CEP: 03563-340. São Paulo-SP, Brasil

Email: brunafonteslara@gmail.com 